\title{
“Microstructured Nanostructures" - Nanostructuring by Means of Conventional Photolithography and Layer-expansion Technique
}

Johannes Platen ${ }^{1}$, Arshak Poghossian ${ }^{1,2, *}$ and Michael J. Schöning ${ }^{1,2}$

${ }^{1}$ Aachen University of Applied Sciences, Jülich Campus, Laboratory for Chemical Sensors and Biosensors, Ginsterweg 1, 52428 Jülich, Germany

${ }^{2}$ Institute of Thin Films and Interfaces (ISG-2), Research Centre Jülich GmbH, 52425 Jülich, Germany

* Author to whom correspondence should be addressed. Email: a.poghossian@fz-juelich.de Received: 28 August 2005 / Accepted: 4 January 2006 / Published: 7 April 2006

\begin{abstract}
A new and simple method for nanostructuring using conventional photolithography and layer expansion or pattern-size reduction technique is presented, which can further be applied for the fabrication of different nanostructures and nano-devices. The method is based on the conversion of a photolithographically patterned metal layer to a metal-oxide mask with improved pattern-size resolution using thermal oxidation. With this technique, the pattern size can be scaled down to several nanometer dimensions. The proposed method is experimentally demonstrated by preparing nanostructures with different configurations and layouts, like circles, rectangles, trapezoids, "fluidic-channel"-, “cantilever"- and meander-type structures.
\end{abstract}

Keywords: Nanostructuring, layer expansion, pattern-size reduction, self-aligned patterning. 


\section{Introduction}

The development of new and easy techniques for the fabrication of nanostructures has been of great interest not only as a possibility for increasing the device-packing density and reducing power consumption, but also for the creation of a new class of nanoelectronic devices, like single-electron transistors [1] or metal/insulator tunnel transistors [2,3], which are based on physico-chemical phenomena that occur on the nano-scale level. Moreover, a coupling of biorecognition elements on nanostructures might allow a creation of functional hybrid systems with a molecular-scale proximity between the molecular recognition and transduction element. Such functional hybrid systems (i.e., the "marriage” of biomolecules and nanostructures) are essential not only for a new generation of chemical and biological nano-sensors with unique functional and application possibilities, but also for the fundamental research of biological molecules (DNA, RNA, immunospecies, proteins, etc.) and living cells.

At present, the minimum size of $\mathrm{Si}$ and GaAs devices is down to $0.25 \mu \mathrm{m}$ or even less. Current CMOS (complementary metal-oxide-semiconductor field-effect transistor) technology will be extended at least to a $50 \mathrm{~nm}$ generation by the year 2012 [4] and a minimum transistor channel length of 20-22 nm is predicted for the year 2014 [5]. Although the resolution of optical lithography continuously increases, to date, for the fabrication of structures below $100 \mathrm{~nm}$, different complicated, cost-intensive and low-throughput techniques (electron- or ion-beam lithography, scanning tunnel or atomic force microscopy) are often utilised [4]. Therefore, recently, some non-conventional techniques in combination with conventional photolithography have been proven for the preparation of nanopattern mask and nano-scale structures. These techniques include a controlled size-reduction using the oxidation of Si [6] or laser-assisted electrochemical etching [7], a chemical-mechanical polishing [8], a decrease of separation between the metallic electrodes by means of an electro-deposition from an electrolyte solution [9], methods that utilise a sidewall structure [5,10,11], a self-aligned plasma etching of a silicon dioxide layer and a silicon substrate [12], a resist thermal reflow and shrinking technique [13], or a lateral partially anodic oxidation of the side-edge of photolithographically structured metallic films (e.g., Ti) in electrolyte solution [14-16], etc.

As an alternative, a new and simple method for the preparation of self-aligned nanostructures using conventional photolithography and layer expansion or pattern-reduction techniques has recently been proposed by the authors [17]. The method was experimentally demonstrated by exemplarily preparing a nano-gap between two "cantilever"-type structures [18]. For this type of structure, the pattern-size of the photolithographically patterned original layer was reduced by a factor of more than 3 [18]. In this work, the feasibility of the proposed method for the preparation of nanostructures with different configurations and layouts such as circles, rectangles, trapezoids, "fluidic-channel”-, "cantilever"- and meander-type structures is studied. In addition, we will also demonstrate that by using the layerexpansion technique, the pattern size can be scaled down to several nanometer dimensions. 


\section{Method}

The proposed nanostructuring method combines the conventional photolithography and the layer expansion or pattern-size reduction technique, which is based on a complete conversion of a photolithographically patterned metal layer to a metal-oxide mask using the thermal oxidation process. Fig. 1 schematically shows the technological process steps for the fabrication of a nano-scaled metaloxide mask and nanostructures according to the proposed method. At first, the metal layer (exemplary $\mathrm{Ta}$ ) is deposited onto the substrate by using a conventional microfabrication technique (Fig. 1(a)) and then, structured using conventional photolithography to form the desired metal pattern with a patternsize resolution, $\mathrm{a}_{\mathrm{ph}}$, defined by the available photolitographic technique (Fig. 1(b)). Then, the metal layer is converted to a metal-oxide $\left(\mathrm{Ta}_{2} \mathrm{O}_{5}\right)$ layer by thermal oxidation, resulting in an expansion of the layer volume and a reduction of the pattern size. In this way, a self-aligned metal-oxide mask with a pattern size $\left(\mathrm{a}_{\mathrm{ox}}<\mathrm{a}_{\mathrm{ph}}\right)$ below the photolithographic resolution is formed (Fig. 1(c)). By a given resolution of the lithographically patterned metal layer, the pattern size of the metal-oxide mask, $a_{0 x}$, will be determined by the thickness of the metal layer $\left(\mathrm{d}_{\mathrm{m}}\right)$ and its ability to expand as it is converted to the respective metal oxide. Metals with a relatively low oxidation temperature and a high ability to expand by conversion to the metal-oxide (e.g., Ta, Ti, etc.) are more suitable as materials for such a metal layer.
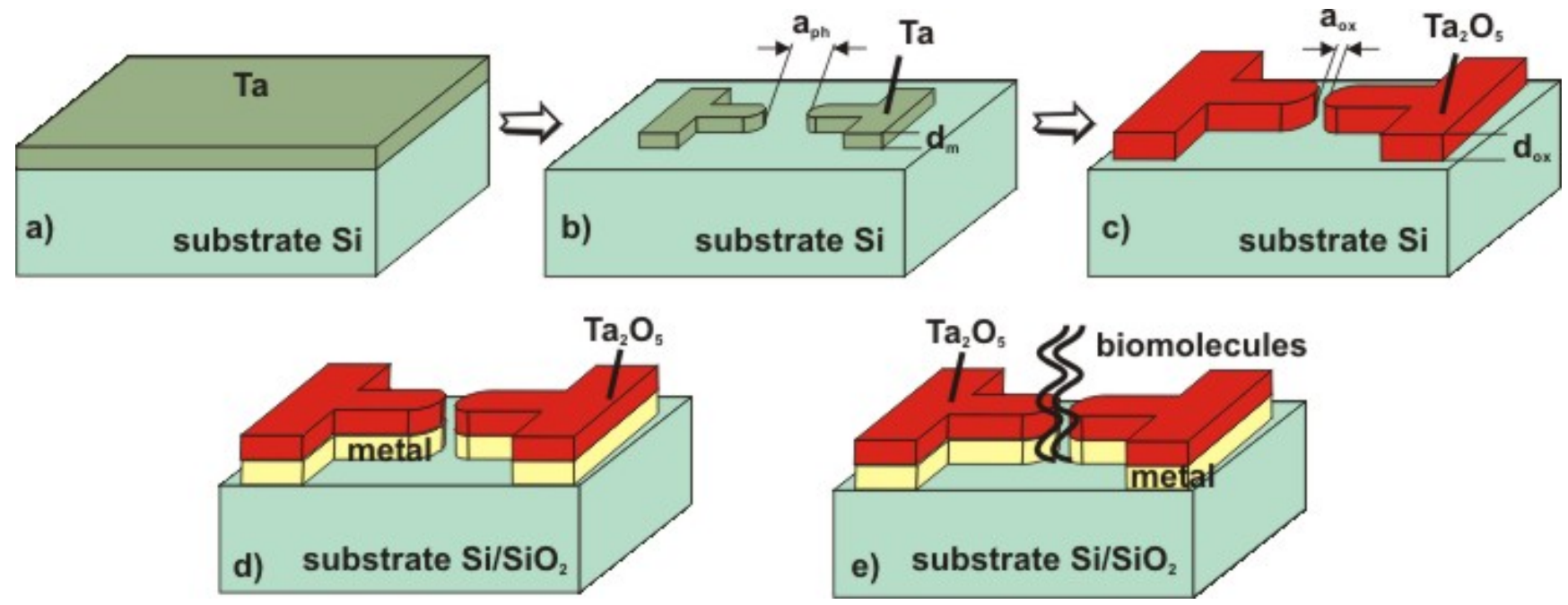

Figure 1. Schematic of the technological process steps for the fabrication of nanostructures: a) deposition of the metallic layer (Ta); b) patterning of the metallic layer with a pattern-size resolution $\left(\mathrm{a}_{\mathrm{ph}}\right)$ of photolithography; c) conversion of the metallic pattern to a metal-oxide layer by thermal oxidation, resulting in a formation of a self-aligned metal-oxide mask with a pattern size $\left(\mathrm{a}_{\mathrm{ox}}\right)$ below the photolithographic resolution; d) nano-gap between two metallic electrodes; e) nano-biosensor by coupling of biomolecules onto the already prepared nanostructure.

Thus, by using this technique, dependent on the thickness of the metal layer and its ability to expand as it is converted to the respective metal oxide, the pattern-size of the original photolitographically patterned structure can be scaled down to several nanometer dimensions. The obtained metal-oxide pattern may then, be used as a mask to realise different nanostructures and nano- 
devices, e.g., nano-slits, nano-trenches, nano-gaps, nano-electrodes, nano-fluidic channels, nano-wires, single-electron transistors, organic field-effect transistors, etc. As an example, Fig. 1(d) schematically shows a nano-gap between two metallic electrodes. In this case, the metal-oxide mask is formed onto the electrode material having a much higher oxidation temperature than that of the patterned metal layer of the mask. Then, the electrode layer is etched using the metal-oxide mask to form a nanometerscaled gap between the two metal electrodes. In addition, different (bio-)chemical nano-sensors can be realised by coupling or immobilising various chemical and biological recognition elements onto the already prepared nanostructures as it is schematically shown in Fig. 1(e). Moreover, this technique is very simple and more realistic to obtain nanometer-scale structures over a large area and with different layouts at both the laboratory and mass-production level.

\section{Experimental}

In this study, Ta has been chosen for the fabrication of the metal-oxide mask due to the relatively low oxidation temperature of $\mathrm{Ta}\left(450-600^{\circ} \mathrm{C}\right)$, the high ability to expand by conversion to the metal oxide (the original thickness of the Ta layer is increased more than 2-2.5 times as it is converted to $\mathrm{Ta}_{2} \mathrm{O}_{5}$ by thermal oxidation [19-21]) and the high chemically resistant properties of $\mathrm{Ta}_{2} \mathrm{O}_{5}$ [22-24]. On the other hand, the well-known high $\mathrm{pH}$-sensitive properties of $\mathrm{Ta}_{2} \mathrm{O}_{5}$ films [21,25-27] are very useful for the further development of different (bio-)chemical nano-sensors.

Ta films of different thicknesses from 200 to $500 \mathrm{~nm}$ have been deposited onto a p-Si substrate by means of electron-beam evaporation or sputtering techniques and then, are patterned by photolithography using a specially designed mask (AutoCAD program was used for the mask development). In order to test the feasibility of the proposed technique for the fabrication of nanostructures with different configurations, the mask layout involves structures with different sizes and configurations (circles, squares, rectangles, trapezoids, meander-shaped structures, "fluidicchannel”- and “cantilever"-type configurations). A lift-off technique or RIE process (using a sulfur hexafluoride $\mathrm{SF}_{6}$ ) has been used to pattern the Ta layer according to the mask layout. Finally, the patterned Ta layers were oxidised at a temperature of $510-517^{\circ} \mathrm{C}$ in dry $\mathrm{O}_{2}$ atmosphere to form the self-aligned $\mathrm{Ta}_{2} \mathrm{O}_{5}$ mask. The investigations of the effectivity (degree) of the expansion of the patterned Ta structures, dependent on the oxidation time, have showed [18] that at an oxidation temperature of $510-530^{\circ} \mathrm{C}$ and an oxidation time of about $2.5-3 \mathrm{~h}$ is sufficient to completely oxidise a $500 \mathrm{~nm}$ Ta layer. The patterned structures have been characterised before the oxidation and after oxidation by means of scanning electron microscopy (SEM).

\section{Results and discussions}

Fig. 2 exemplarily presents an SEM picture of a prepared nano-slit. For comparison, the SEM picture of the slit before the oxidation is presented, too. The conversion of the Ta layer to the $\mathrm{Ta}_{2} \mathrm{O}_{5}$ layer by thermal oxidation results in an expansion of the layer volume, including the lateral expansion and hence, to a decrease in the width of the slit. After oxidation of the patterned $500 \mathrm{~nm}$ thick Ta layer at $515^{\circ} \mathrm{C}$ for about $3 \mathrm{~h}$, the original (photolitographically patterned) slit of $1420 \mathrm{~nm}$ in width has been reduced down to $330 \mathrm{~nm}$. Thus, after oxidation, the pattern-size resolution was improved for about 
$1100 \mathrm{~nm}$ or by a factor of more than 4. By applying even thicker Ta layers or photolitographically patterned structures with a pattern size of less than $\sim 1 \mu \mathrm{m}$, slits with a width of a few nanometer can be formed by using this technique.

Fig. 3 shows SEM photographs of a circle-type structure before (a) and after (b) oxidation of the photolithographically patterned Ta layer. The diameter of the circle before the oxidation was $1230 \mathrm{~nm}$. After Ta oxidation, a circle-like structure has been obtained with a diameter of $620 \mathrm{~nm}$. Currently, with a circle-type configuration, "only" a pattern-size reduction by the factor of about 2 has been achieved, which is smaller than that obtained for other configurations studied in this work. In addition, the obtained structure has not an exactly uniform circle-shaped configuration. A possible explanation for such a behavior could be a competition expansion of the Ta layer during the oxidation process along the different radial directions parallel to the surface of the circular structure.
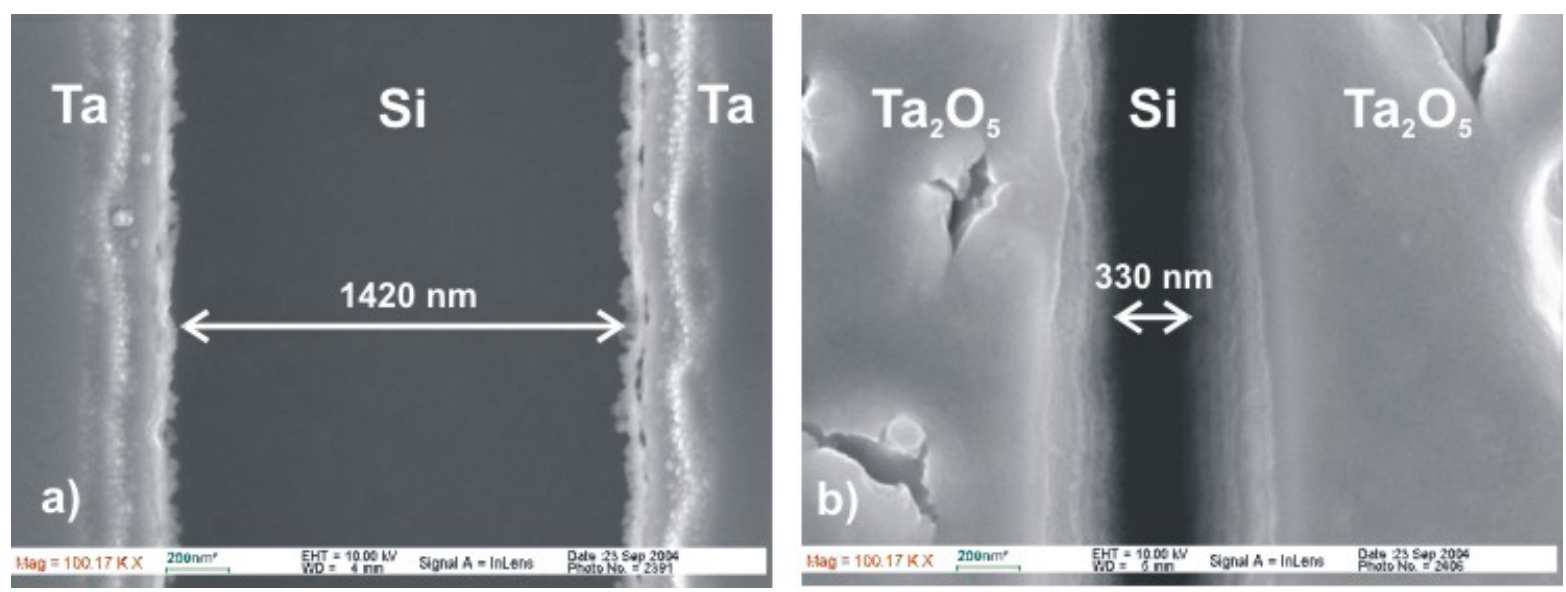

Figure 2. Scanning electron microscopy picture of prepared nano-slit before (a) and after (b) Ta oxidation. A pattern-size reduction of about $1100 \mathrm{~nm}$ or by a factor of more than 4 has been achieved.
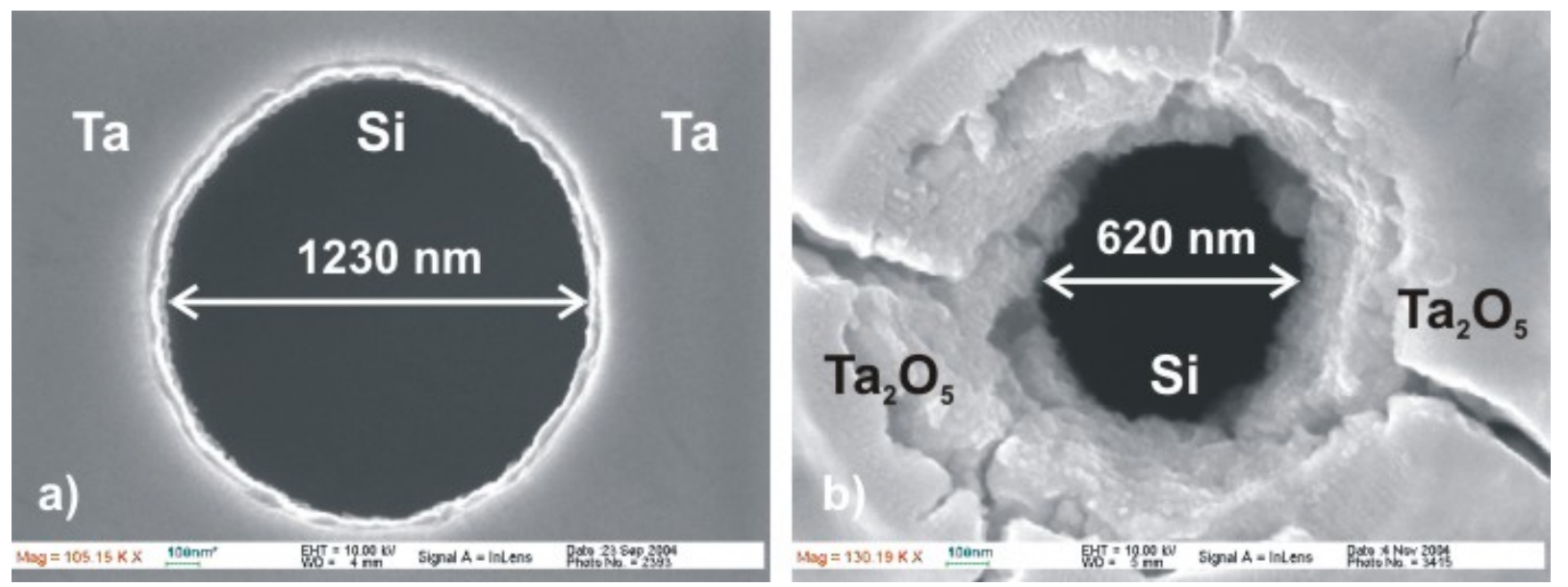

Figure 3. Scanning electron microscopy picture of a prepared circle-shaped structure before (a) and after (b) Ta oxidation. A pattern-size reduction of about $610 \mathrm{~nm}$ or by a factor of about 2 has been achieved.

Best results have been obtained with a so-called "fluidic-channel”-type structure (see Figs. 4 and 5). The original width of the structure before the oxidation of the photolithography patterned Ta layer to 
$\mathrm{Ta}_{2} \mathrm{O}_{5}$ was $1420 \mathrm{~nm}$ (Fig. 4(a)). After oxidation of the patterned $500 \mathrm{~nm}$ thick Ta layer at $515^{\circ} \mathrm{C}$ for about $2.5 \mathrm{~h}$, the resulting width of the structure was $120 \mathrm{~nm}$ (Fig. 4(b)). Thus, after oxidation, the pattern-size was reduced for about $1300 \mathrm{~nm}$ or by a factor of about 12 . As it can be seen from Fig. 5, with this "fluidic-channel"-type configuration, after oxidation even structures below $10 \mathrm{~nm}$ are possible to prepare. Thus, a pattern-size reduction by a factor of more than 100 can be achieved. However, the degree of lateral expansion during the conversion of the Ta layer to $\mathrm{Ta}_{2} \mathrm{O}_{5}$ was not sufficiently uniform. As it can be seen from Figs. 4 and 5, a large expansion has been observed in the middle regions in comparison to the edges of the structure. The reasons for this effect are not yet clear in detail.

a)

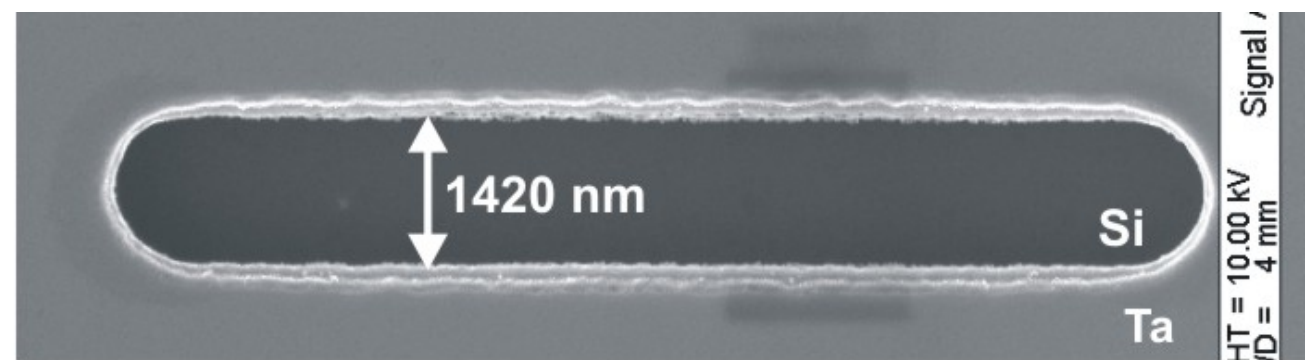

b)

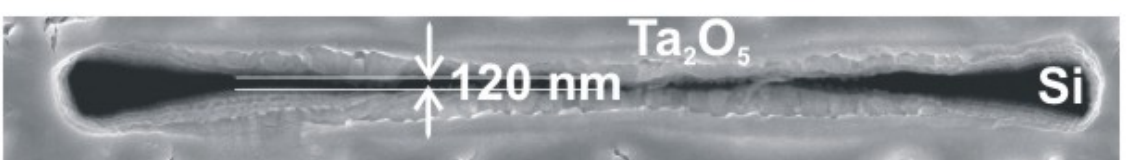

Figure 4. Scanning electron microscopy picture of a prepared "fluidic-channel"-type structure before (a) and after (b) Ta oxidation. A pattern-size reduction of about $1300 \mathrm{~nm}$ or by a factor of about 12 has been achieved.

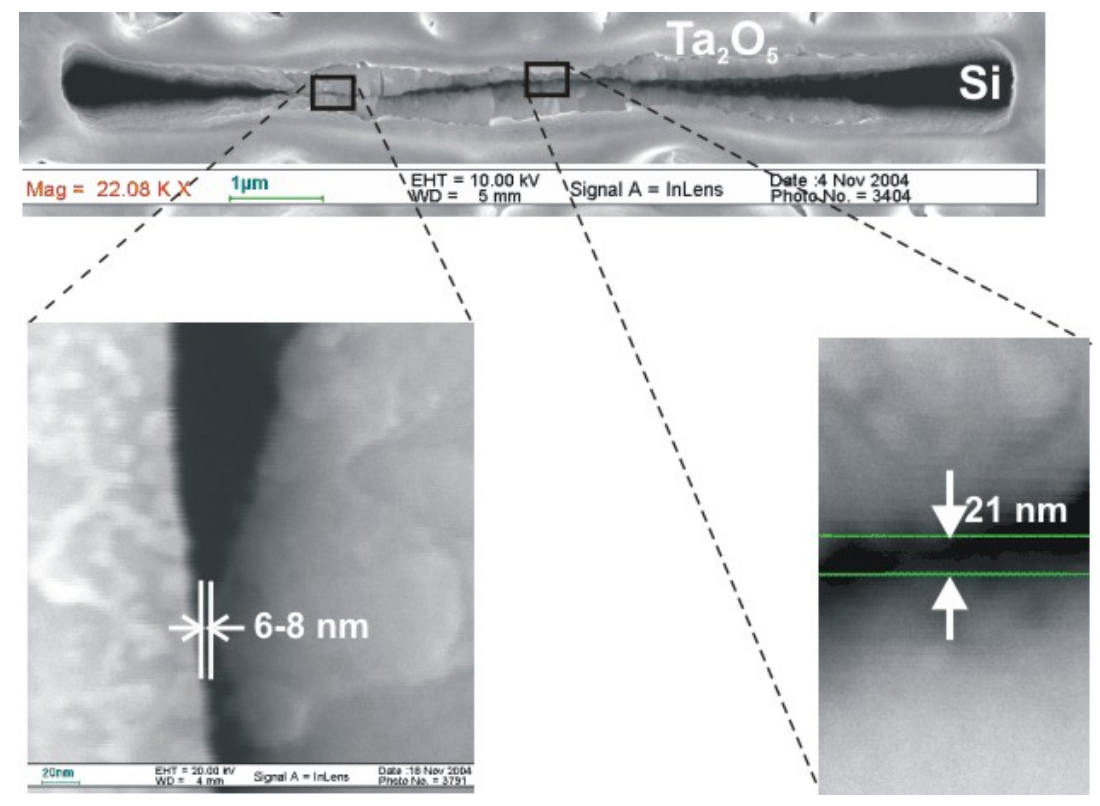

Figure 5. Experimental demonstration of feasibility of the proposed method for the preparation of even structures below $10 \mathrm{~nm}$. 


\section{Conclusions}

A new method for the preparation of self-aligned nanostructures by combining conventional photolithography and layer expansion or pattern-size reduction technique has been proposed and realised. The method is based on a complete conversion of a photolithographically patterned metal layer to a metal-oxide mask with improved pattern-size resolution using the thermal oxidation process. By applying this technique, dependent on the thickness of the metal layer and its ability to expand, the pattern-size resolution of conventional photolithography can be scaled down to nanometer dimensions. Moreover, this technique is very simple, easy and more realistic to obtain nanometer-scale structures over a large area and with different layouts at both the laboratory and mass-production level. The proposed method has been experimentally demonstrated by preparing nanostructures with different configurations and layouts. Moreover, the feasibility of this method for the preparation of even structures below $10 \mathrm{~nm}$ has been experimentally validated. Nonetheless, due to a possible competition expansion of the Ta layer during the oxidation process along different directions parallel to the surface, the obtained nanostructures are not sufficiently uniform. Therefore, further experiments are needed in order to study the specificity of lateral expansion of the Ta layers of different layouts during the oxidation processes. These investigations might be useful in order to optimise the layouts of the original structures (before oxidation) as well as to obtain the desired or required configuration of the final nanostructures (i.e., after oxidation).

\section{Acknowledgements}

Parts of this work were supported by the Ministerium für Innovation, Wissenschaft, Forschung und Technologie des Landes Nordrhein-Westfalen.

\section{References}

1. Namatsu, H.; Watanabe, Y.; Yamazaki, K.; Yamaguchi, T.; Nagase, M.; Ono, Y.; Fujiwara, A.; Horiguchi, S. Fabrication of Si single-electron transistors with precise dimensions by electronbeam nanolithography. J. Vac. Sci. Technol. B, 2003, 21, 1.

2. Sasajima, R.; Fujimaru, K.; Matsumura, H. A metal insulator tunnel transistor with $16 \mathrm{~nm}$ channel length. Appl. Phys. Lett., 1999, 74, 3215.

3. Fujimaru, K.; Sasajima, R.; Matsumura, H. Nanoscale metal transistor control of FowlerNordheim tunneling currents through 16 nm insulating channel. J. Appl. Phys., 1999, 85, 6912.

4. Chen, Y.; Pepin, A. Nanofabrication: Conventional and nonconventional methods. Electrophoresis, 2001, 22, 187.

5. Horstmann, J.T.; Goser, K.F. New fabrication technique for nano-MOS transistors with W=25 nm and $\mathrm{L}=25 \mathrm{~nm}$ using only conventional optical lithography. Microelectron. Eng., 2002, 61-62, 601.

6. Heidemeyer, H.; Single, C.; Zhou, F.; Prins, F.E.; Kern, D.P.; Plies, E.J. Self-limiting and pattern dependent oxidation of silicon dots fabricated on silicon-on-insulator material. Appl. Phys., 2000, 87,4580 . 
7. Juhasz, R.; Linnros, J. Silicon nanofabrication by electron-beam lithography and laser-assisted electrochemical size-reduction. Microelectron. Eng., 2002, 61-62, 563.

8. Lee, C.-S.; Han, C.-H. A novel sub-micron gap fabrication technology using chemical-mechanical polishing (CMP): Application to lateral field emission device (FED). Sens. Actuators A, 2002, 9798, 739.

9. Morpurgo, A.F.; Marcus, C.M.; Robinson, D.B. Controlled fabrication of metallic electrodes with atomic separation. Appl. Phys. Lett., 1999, 74, 2084.

10. Chung, K.-H.; Sung, S.-K.; Kim, D.H.; Choi, W.Y.; Lee, C.A.; Lee, J.D.; Park, B.-G. Nanoscale multi-line patterning using sidewall structure. Jpn. J. Appl. Phys., 2002, 41, 4410.

11. Choi, Y.-K.; Zhu, J.; Grunes, J.; Bokor, J.; Somorjai, G.A. Fabrication of sub-10-nm silicon nanowire arrays by size reduction lithography. J. Phys. Chem. B 2003, 107, 3340.

12. Georgiev, G.; Müller-Wiegand, M.; Georgieva, A.; Ludolph, K.; Oesterschulze, E. Lithographyfree fabrication of sub-100 nm structures by self-aligned plasma etching of silicon dioxide layers and silicon. J. Vac. Sci. Technol. B, 2003, 21, 1361.

13. Meng, C.C.; Liao, G.R.; Lu, S.S. Formation of submicron T-gate by rapid thermally reflowed resist with metal transfer layer. Electron. Lett., 2001, 37, 1045.

14. Hashioka, S.; Matsumura, H. 10-nm-size fabrication of semiconductor substrates and metal thin lines by conventional photolithography. Jpn. J. Appl. Phys., 2000, 39, 7063.

15. Fujimaru, K.; Ono, T.; Nagai, R.; Matsumura, H. Nanometer pattern-mask fabricated by conventional photolithography. Jpn. J. Appl. Phys., 1997, 36, 7786.

16. Hashioka, S.; Mogi, T.; Matsumura, H. Novel nano-fabrication technique with low edge roughness. Jpn. J. Appl. Phys., 2003, 42, 4169.

17. Poghossian, A.; Schöning, M.J. German patent application, DE 1033275.8, 2003.

18. Poghossian, A.; Schöning, M.J.; Platen, J. Towards self-aligned nanostructures by means of layer expansion technique. Electrochim. Acta, 2005, 51, 838.

19. Atanassova, E.; Spassov, D. Electrical properties of thin $\mathrm{Ta}_{2} \mathrm{O}_{5}$ films obtained by thermal oxidation of Ta on Si. Microelectron. Reliability, 1998, 38, 827.

20. Park, S.W.; Im, H.B. Effect of oxidation conditions on the properties of tantalum oxide-films on silicon substrates. Thin Solid Films, 1992, 207, 258.

21. Poghossian, A.; Schöning, M.J. Detecting both physical and (bio-)chemical parameters by means of ISFET devices. Electroanalysis, 2004, 16, 1863.

22. Christensen, C. ; de Reus, R.; Bouwstra, S. Tantalum oxide thin films as protective coatings for sensors. J. Micromech. Microeng., 1999, 9, 113.

23. Chaneliere, C. ; Autran, J.L.; Devine, R.A.B.; Balland, B. Tantalum pentoxide $\left(\mathrm{Ta}_{2} \mathrm{O}_{5}\right)$ thin films for advanced dielectric applications. Mater. Sci. Eng., 1998, R22, 269.

24. Schöning, M.J.; Brinkmann, D.; Rolka, D.; Demuth, C.; Poghossian, A. CIP (cleaning-in-place)suitable "non-glass" pH sensor based on a $\mathrm{Ta}_{2} \mathrm{O}_{5}$-gate EIS structure. Sens. Actuators B, 2005, 111112, 423.

25. Poghossian, A.; Baade, A.; Emons, H.; Schöning, M.J. Application of ISFETs for $\mathrm{pH}$ measurement in rain droplets. Sens. Actuators B, 2001, 76, 634. 
26. Kwon, D.-H.; Cho, B.-W.; Kim, C.-S.; Sohn, B.-K. Effects of heat treatment on $\mathrm{Ta}_{2} \mathrm{O}_{5}$ sensing membrane for low drift and high sensitivity pH-ISFET. Sens. Actuators B, 1996, 34, 441.

27. Hara, H.; Ohta, T. Dynamic response of a $\mathrm{Ta}_{2} \mathrm{O}_{5}$-gate $\mathrm{pH}$-sensitive field-effect transistor. Sens. Actuators B, 1996, 32, 115.

(C) 2006 by MDPI (http://www.mdpi.org). Reproduction is permitted for non-commercial purposes. 